\title{
Injectable and Sprayable Polyphenol-Based Hydrogels for Controlling Hemostasis
}

Huimin Geng,,$^{\dagger}$ Qiong Dai,$^{\dagger}$ Haifeng Sun,$^{\dagger}$ Liping Zhuang, ${ }^{\dagger}$ Aixin Song, ${ }^{\dagger}$ Frank Caruso, ${ }^{\dagger, \S}$ Jingcheng Hao, ${ }^{\dagger}$ Jiwei Cui ${ }^{\dagger, \dagger, *}$

†Key Laboratory of Colloid and Interface Chemistry of the Ministry of Education,

School of Chemistry and Chemical Engineering, Shandong University, Jinan, Shandong 250100, China

${ }^{\ddagger}$ State Key Laboratory of Microbial Technology, Shandong University, Qingdao, Shandong 266237, China

${ }^{\S}$ ARC Centre of Excellence in Convergent Bio-Nano Science and Technology, and the Department of Chemical Engineering, The University of Melbourne, Parkville, Victoria 3010, Australia

KEYWORDS: Polyphenol; Tannic acid; Injectable hydrogel; Hemostasis; Selfhealing 
ABSTRACT: Injectable and sprayable hydrogels have attracted considerable attention for application in the biomedical field owing to their high moldability and efficiency in encapsulating therapeutics and cells. Herein, we report the spontaneous assembly of injectable and sprayable hydrogels via a one-step mixing of solutions of tannic acid (TA) and O-carboxymethyl chitosan (CMCS) without an external stimulus. The presence of 1,4-benzenediboronic acid (BDBA) improves the mechanical properties and reduces the gelation time of the resulting hydrogels. The hydrogels assemble via hydrogen bonds between TA and CMCS as well as via dynamic boronate ester bonds between TA and BDBA, as confirmed by Fourier Transform-Infrared spectroscopy. Balancing the interactions between the three components (CMCS/TA/BDBA) is essential for the construction of the hydrogels. The moduli of the CMCS-TA-BDBA hydrogels initially increased as the amount of BDBA increased and decreased after reaching a maximum at a BDBA-to-TA molar ratio of 3:1. The CMCS-TA-BDBA hydrogels with interconnected porous morphologies display rapid gelation ( 10 s), biocompatibility, and self-healing, injectable and sprayable abilities. In addition, the hydrogels can be used for hemostasis. The extent of bleeding in mouse livers treated with the hydrogels could be reduced extensively from 240 (non-treated mouse livers) to $55 \mathrm{mg}$ (77\% reduction). The reported hydrogels coupled with the combination of functionality and biological activity make them promising hemostatic materials for biomedical applications. 


\section{INTRODUCTION}

Hydrogels have numerous common physiochemical properties to those of the natural extracellular matrix (e.g., elasticity and low interfacial tension) and can be shaped into various forms including membranes, sheets, or coatings. ${ }^{1-3}$ In the biomedical field, hydrogels are of scientific and practical interest owing to their flexibility in fabrication, good biocompatibility, and desirable physiochemical characteristics. ${ }^{4,5}$ For instance, injectable hydrogels that can be injected through a small pinhead to alleviate the pain of patients have been widely studied. ${ }^{2,5,6}$ In contrast, to date, fewer studies have focused on sprayable hydrogels, which are formed instantly by spraying two liquid precursors. ${ }^{7}$ Injectable and sprayable hydrogels are convenient to use and have advantages (e.g., suitable for tissue surfaces with a complex and non-flat geometry, high-efficiency in encapsulation and protection of therapeutic agents) in biomedical and pharmaceutical applications such as postsurgical cancer treatment. ${ }^{8}$ To ensure injectability and sprayability, the system should be either in a solution or have shear thinning properties before practical use, and gelation should then occur rapidly via either chemical crosslinking or physical association. ${ }^{1,6}$ In situ chemical cross-linking is a conventional approach used to prepare hydrogels, which is generally achieved by free radical polymerization, Schiff base reaction, or Michael addition. ${ }^{1,9,10}$ However, the residue initiators or in vivo chemical reaction of these processes can restrict the application of such hydrogels as implanted biomaterials. ${ }^{1,11}$ Alternatively, physical gelation can be a more suited approach, wherein an important gel-inducing factor is the human body temperature. Well-known thermosensitive hydrogels based on $\operatorname{poly}(N-$ 
isopropylacrylamide) and polyester have been thoroughly studied.,12 Furthermore, physical hydrogels derived from molecular self-assembly offer new opportunities for the design and fabrication of biomimetic materials. They have additional advantages of being highly biocompatible, degradable, and be easily metabolized. ${ }^{4}$ However, it is challenging to achieve rapid gelation in the absence of external stimuli, as well as a combination of functionality and biological activity in self-assembled hydrogels.

Hydrogels with three-dimensional (3D) networks and high mechanical strength can absorb wound discharge and provide a moist environment for wound healing. ${ }^{13,14}$ For instance, hydrogels based on various active components have been designed as hemostats, bacteriostats, and tissue adhesives for wound closure. ${ }^{15,16}$ Specifically, naturally derived polyphenols, such as tannic acid (TA), have been found to exhibit antioxidant, antibacterial, and anti-inflammatory effects, and have been widely used for designing multifunctional nanoparticles or films for different biomedical applications. ${ }^{17-20}$ Recently, it has been reported that TA can also act as a hemostatic adhesive, owing to the interactions between blood and the phenolic hydroxyl groups of TA, leading to faster blood clotting. ${ }^{21,22}$ The negatively charged polyphenol acted as a contact activator and could thus activate clotting factor XII in the body, which improved the hemostatic capability of phenolic materials. ${ }^{22,23}$ TA can also be used as a molecular cross-linker to interact with synthetic polymers and biomacromolecules, including polyvinylpyrrolidone,${ }^{24}$ polyethylene glycol (PEG),${ }^{25}$ polypyrrole, ${ }^{26}$ thrombin,${ }^{27}$ and gelatin, ${ }^{28}$ via hydrogen bonding and hydrophobic interactions. Furthermore, polyphenols can interact with metal ions (e.g., $\left.\mathrm{Fe}^{3+}\right)$ and boron ions $\left(\mathrm{B}^{3+}\right)$ to form $\mathrm{pH}-$ 
responsive dynamic covalent bonds. ${ }^{29,30}$ Therefore, TA is a versatile candidate for applications in materials science and biotechnology. ${ }^{30,31}$ However, most polymer-TA complexes in water do not form hydrogels, unless in the presence of additives or external stimuli, thus often leading to either homogeneous solutions or coacervation. ${ }^{24}$ TA-based hydrogels have been mostly reported as membrane-like gels generated via layer-by-layer assembly, ${ }^{33,34}$ which requires an oxidation initiator (e.g., $\mathrm{FeCl}_{3}$ ) and $\mathrm{pH}$ adjustments for gelation to occur. ${ }^{24,35}$ Therefore, precise control over the interactions between polymers and TA is important for the development of high-performance TAbased multifunctional hydrogels.

Herein, we report a facile and rapid strategy to fabricate injectable and sprayable hydrogels based on TA and O-carboxymethyl chitosan (CMCS). Water-soluble CMCS is nontoxic, biodegradable, hemostatic, antibacterial, and antifungal, thereby endowing functionalities to the final hydrogels. ${ }^{22}$ TA served as a "molecular glue" to cross-link CMCS into a network. The addition of 1,4-benzenediboronic acid (BDBA) improved the mechanical properties and reduced the gelation time of the hydrogels (Scheme 1). For example, when compared with the hydrogel prepared in the absence of BDBA, the CMCS-TA-BDBA hydrogel displayed a higher storage modulus of $2.5 \times 10^{4} \mathrm{~Pa}$ at 10 $\mathrm{Hz}(\mathrm{cf} 7.2 \mathrm{~Pa})$ and a faster gelation time of $10 \mathrm{~s}(\mathrm{cf} 1 \mathrm{~min})$. Optimization of hydrogel preparation was achieved by controlling the concentration and ratio of the components. The combination of CMCS, TA, and BDBA provides a rapid and versatile approach to engineer biocompatible hydrogels with injectable and sprayable abilities for potential applications in hemostasis. 
Scheme 1. Scheme of the formation of the CMCS-TA-BDBA hydrogel and the interactions between CMCS, TA and BDBA. The vial in the inset is a photograph of the CMCS-TA-BDBA hydrogel.

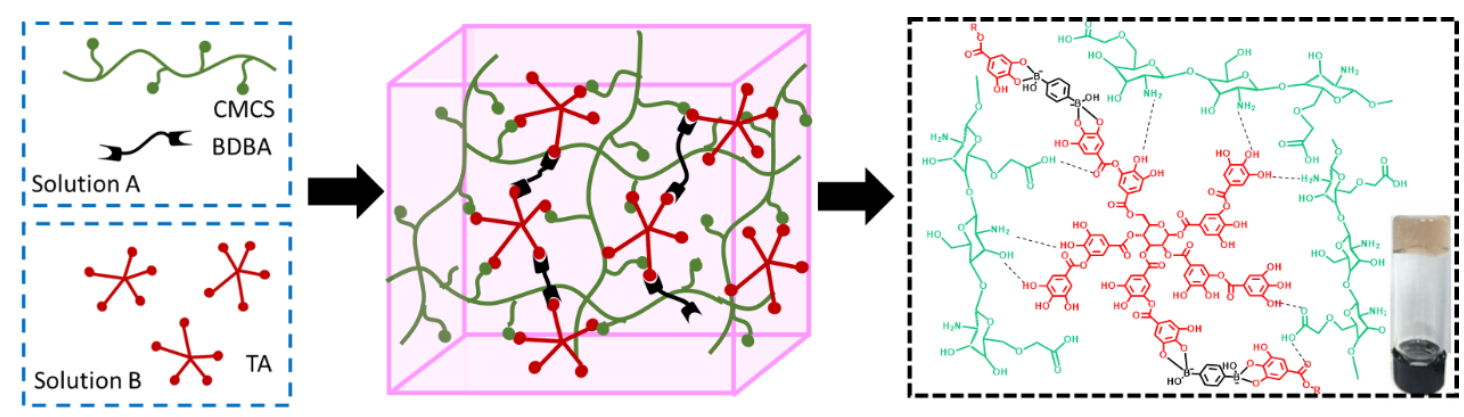

\section{MATERIALS AND METHODS}

Materials. CMCS $(M \mathrm{w} 20-30 \mathrm{kDa}$, degree of carboxylation $\approx 80 \%)$ was obtained from Beijing Solarbio Science \& Technology Co., Ltd. (Beijing, China). TA and BDBA were purchased from J\&K Scientific Ltd. (Beijing, China). Rhodmine B and methylene blue were purchased from Fuchen Chemical Reagent Co., Ltd. (Tianjin, China). These reagents were used without any further purification.

Preparation of CMCS-TA and CMCS-TA-BDBA Hydrogels. For the preparation of the CMCS-TA hydrogels, precursor solutions A and B were first respectively prepared by dissolving CMCS and TA in water at desired concentrations and mixed subsequently. Specifically, CMCS was dissolved in water to achieve a concentration of $60 \mathrm{mg} \mathrm{mL}^{-1}$ (precursor solution A). Precursor solution B was prepared by dissolving TA in water to achieve a concentration of $40 \mathrm{mg} \mathrm{mL}^{-1}$. Subsequently, $0.5 \mathrm{~mL}$ of solution B was added dropwise into $0.5 \mathrm{~mL}$ of solution A under vortex, resulting in the instant formation of the CMCS-TA hydrogel. 
For the preparation of the CMCS-TA-BDBA hydrogels, precursor solution A consisted of a solution of CMCS and BDBA in water, wherein $0.1 \mathrm{M} \mathrm{NaOH}$ was used to aid solubilization (the final $\mathrm{pH}$ was regulated at 9.0). Typically, the CMCS-TA-BDBA hydrogels were obtained upon mixing of $0.5 \mathrm{~mL}$ of solution A, containing CMCS (60 $\left.\mathrm{mg} \mathrm{mL}{ }^{-1}\right)$ and BDBA $(0.96 \mathrm{mg})$, and $0.5 \mathrm{~mL}$ of solution B containing TA $\left(20 \mathrm{mg} \mathrm{mL}^{-1}\right)$; the ratio of BDBA and TA was 1:1. CMCS-TA-BDBA hydrogels at other BDBA-toTA ratios (3:1) were also prepared.

Self-Healing of Hydrogels. To macroscopically examine the self-healing properties of the hydrogels, the CMCS-TA hydrogels were stained with either rhodamine B or methylene blue and sectioned into halves. Two halves with different colors were combined into an integral hydrogel. After incubation in a petri dish for $1 \mathrm{~min}$, the hydrogels were suspended under gravity.

Injectability of Hydrogels. CMCS-TA-BDBA hydrogels (BDBA/TA 1:1) stained with rhodamine B were introduced into a syringe fitted with a $26 \mathrm{G}$ needle and then injected onto a glass slide. In a separate experiment, equivalent volumes of precursor solution A containing rhodamine B and precursor solution B were loaded into separate syringes ( $5 \mathrm{~mL}$ capacity) within a dual-syringe applicator. The solutions were extruded through a "Y" shape connector and then through a narrow needle (20 G), finally forming hydrogels at room temperature $\left(25^{\circ} \mathrm{C}\right)$.

Sprayabiliy of Hydrogels. The gelation of the CMCS-TA-BDBA hydrogels (BDBA/TA 3:1) was also examined in sprayable devices. Precursor solutions A and B were separately loaded into $5 \mathrm{~mL}$ sprayable bottles. The two liquid precursors were 
then sprayed onto a petri dish either in sequence or simultaneously, leading to the spontaneous formation of a hydrogel film.

Morphological Observation. Scanning electron microscopy (SEM) images were obtained from a Zeiss G300 field-emission scanning electron microscope (Germany). freeze-dried hydrogels were sputtered with gold for $180 \mathrm{~s}$ at $2 \mathrm{~mA}$ before observation. Rheological Characterization. The mechanical properties of the hydrogels were examined using an RS6000 rheometer (HAAKE) with a cone-plate system $\left(\mathrm{C} 35 / 1^{\circ} \mathrm{Ti}\right.$ L07116, diameter $35 \mathrm{~mm}$, and core angle $\left.1^{\circ}\right)$. The storage modulus $\left(G^{\prime}\right)$ and loss modulus $\left(G^{\prime \prime}\right)$ of the hydrogels were measured by frequency sweeps $(0.1-100 \mathrm{~Hz})$ at a strain level of $1 \%$. The self-healing properties of the hydrogels were investigated by straining the gels under an alternately changing amplitude of oscillatory force from small stress $(1 \mathrm{~Pa})$ to large stress $(800 \mathrm{~Pa})$ at fixed intervals of $100 \mathrm{~s}$. The experiments were performed at $25^{\circ} \mathrm{C}$.

In Vitro Cytotoxicity Assay. The in vitro cytotoxicity of the hydrogels was evaluated by a methylthiazolyl-diphenyl-tetrazolium bromide (MTT) assay using MCF-7 human breast tumor cells. Degradation of the hydrogels was performed by immersing $1 \mathrm{~g}$ of the hydrogels in $10 \mathrm{~mL}$ of $0.2 \mathrm{M} \mathrm{NaOH}$ solution for $24 \mathrm{~h}$. The $\mathrm{pH}$ of the solution was adjusted to 7.4 , and the solution was filtered through a $0.22 \mu \mathrm{m}$ filter. MCF-7 cells were suspended in $200 \mu \mathrm{L}$ cell culture medium and seeded into 96-well plates at a density of $1 \times 10^{4}$ cells/well and incubated for $24 \mathrm{~h}$. Then, the solution within which the hydrogels degraded was added to the 96-well plate, and the cells were incubated for another $24 \mathrm{~h}$ prior to MTT assays. 
Hemostatic Ability of Hydrogels. A mouse-hemorrhaging liver model was used for controlling hemostasis studies. ${ }^{36,37}$ The mouse (Kunming mice, weight of 40-50 g, female) was anesthetized prior to surgery. After abdominal incision, the mouse liver was carefully exposed, and a pre-weighed filter paper on a paraffin film was placed beneath the liver. The liver was bled by puncturing with a $23 \mathrm{G}$ needle. The bleeding site was immediately treated with $200 \mu \mathrm{L}$ of CMCS-TA-BDBA (BDBA/TA 1:1) injected hydrogel or preweighed sterile gauze (positive control). No treatment group was considered as a negative control. Blood loss was estimated by weighing the filter paper with adsorbed blood. Animal studies were conducted following the regulations of the Animal Ethics Review of Shandong University and the Health Guide for the Care and Use of Laboratory Animals of National Institutes.

\section{RESULTS AND DISCUSSION}

CMCS is a water-soluble chitosan derivative with a $\mathrm{p} K$ a of $2.0-4.0 .{ }^{38}$ Upon addition of TA solution (40 mg mL $\mathrm{m}^{-1}$ in water, $\left.0.5 \mathrm{~mL}, \mathrm{pH} 3.4\right)$ to CMCS solution $\left(60 \mathrm{mg} \mathrm{mL}^{-1}\right.$ in water, $0.5 \mathrm{~mL}, \mathrm{pH} 9.8$ ), a fast-forming CMCS-TA hydrogel with a neutral $\mathrm{pH}(\mathrm{pH} \sim 7.4)$ was obtained. TA has a $\mathrm{p} K_{\mathrm{a}}$ of $\sim 8.5$ and its phenolic groups act as excellent $\mathrm{H}$ donors. ${ }^{24}$ Therefore, strong hydrogen bonding and electrostatic interactions between TA and CMCS drives the formation of the CMCS-TA hydrogels. By controlling the concentration of CMCS and TA, solutions, gels, or coacervation (phase separation) was observed (Figure S1). Systematically, as depicted in Figure 1a, when the TA concentration was lower than $20 \mathrm{mg} \mathrm{mL}^{-1}$ or higher than $88 \mathrm{mg} \mathrm{mL}^{-1}$, the mixture could not form hydrogels. This might be due to insufficient hydrogen bond formation between 
CMCS and TA to build a gelatinous network structure at low TA concentrations. This result is consistent with the frequency sweeping experiments: at TA concentrations below $20 \mathrm{mg} \mathrm{mL}^{-1}$ (at a given CMCS concentration of $30 \mathrm{mg} \mathrm{mL}^{-1}$ ), $G^{\prime \prime}$ was higher than $G^{\prime}$, which is characteristic of liquid-like materials (Figure 1b). ${ }^{24}$ At TA concentrations higher than $88 \mathrm{mg} \mathrm{mL}^{-1}$, phase separation was observed. Thus, gel formation was observed within a TA concentration range of $20 \sim 88 \mathrm{mg} \mathrm{mL}^{-1}$ and the gelation time can be tuned from minutes to seconds by changing the TA concentration. It was difficult to prepare homogeneous hydrogels at CMCS concentrations greater than $50 \mathrm{mg} \mathrm{mL} \mathrm{L}^{-1}$ owing to the poor fluidity of CMCS. As expected, hydrogels with weak rheological properties were obtained at low concentrations of CMCS $\left(<20 \mathrm{mg} \mathrm{mL}^{-1}\right)$. These results indicate that the concentrations of TA and CMCS influence their intermolecular interactions and further influence the mechanical properties of the hydrogels. At a given CMCS concentration of $30 \mathrm{mg} \mathrm{mL}^{-1}$ and TA at a concentration range of $20 \sim 50 \mathrm{mg} \mathrm{mL}^{-1}, G^{\prime}$ was higher than $G^{\prime \prime}$, characteristic of an elastic material rather than a viscous material (Figure 1b), ${ }^{39}$ and the hydrogels showed injectable properties. Moreover, at $10 \mathrm{~Hz}, G^{\prime}$ and $G^{\prime \prime}$ increased from 2.5 and $4.9 \mathrm{~Pa}$ to $2.4 \times 10^{3}$ and $2.0 \times 10^{2} \mathrm{~Pa}$, respectively, as the TA concentration increased from 10 to $50 \mathrm{mg}$ $\mathrm{mL}^{-1}$ at a given CMCS concentration of $30 \mathrm{mg} \mathrm{mL}^{-1}$. 

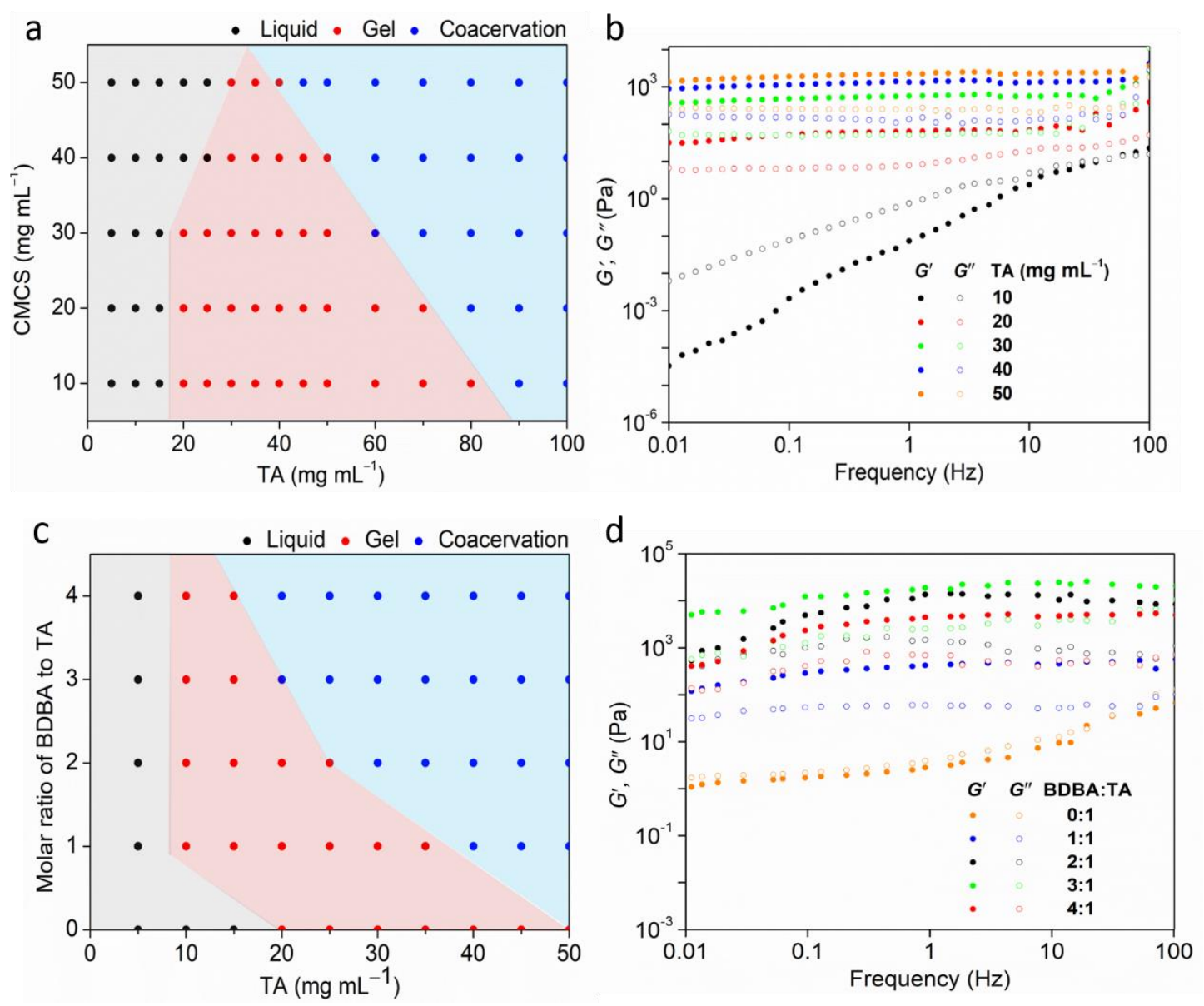

Figure 1. (a) Phase diagrams of mixtures of CMCS and TA. (b) Storage modulus $\left(G^{\prime}\right)$ and loss modulus $\left(G^{\prime \prime}\right)$ of CMCS-TA hydrogels versus frequency. The CMCS concentration was fixed at $30 \mathrm{mg} \mathrm{mL}^{-1}$. (c) Phase diagrams of mixtures of TA and BDBA at a fixed CMCS concentration of $30 \mathrm{mg} \mathrm{mL}^{-1}$. (d) $G^{\prime}$ and $G^{\prime \prime}$ of the CMCSTA-BDBA hydrogels versus frequency. CMCS and TA concentrations were fixed at 30 and $10 \mathrm{mg} \mathrm{mL}^{-1}$, respectively. Frequency sweep was performed at a constant strain of $1 \%$ from 0.01 to $100 \mathrm{~Hz}$ at $25^{\circ} \mathrm{C}$.

It is well known that phenolic compounds easily oxidize to quinones. ${ }^{40}$ Recent studies have demonstrated that boronic acid-based molecules can strongly interact with cis-diol structures to protect them from spontaneous autoxidation under basic conditions. ${ }^{39,40}$ 
Thus, BDBA was introduced into the bicomponent system because it can form tetrahedral boronate esters with the catechol and gallic end-groups of TA (Figure S2). Furthermore, BDBA is stable in biological conditions and displays zero-to-negligible toxicity against the human body. ${ }^{15,42,43}$ Upon addition of BDBA to the CMCS/TA homogeneous solution, the mixture rapidly cross-linked into stable hydrogels, even at a low TA concentration of $10 \mathrm{mg} \mathrm{mL}^{-1}$ (Figure 1c).

To determine the driving forces in hydrogel self-assembly, Fourier transform infrared (FT-IR) spectroscopy was performed (Figure S3). TA and CMCS showed strong, broad bands of $-\mathrm{OH}$ stretching vibration at 3454 and $3428 \mathrm{~cm}^{-1}$, respectively. In the spectrum of the CMCS-TA hydrogel, the -OH vibration characteristic peak shifted to $3400 \mathrm{~cm}^{-1}$, which demonstrates the existence of hydrogen bonds between CMCS and TA. ${ }^{24,44}$ The $-\mathrm{C}=\mathrm{O}$ vibration characteristic peak in TA was observed at $1723 \mathrm{~cm}^{-1}$ and shifted to $1714 \mathrm{~cm}^{-1}$ when the CMCS-TA hydrogel was formed, which suggests that the carbonyl groups were involved in the hydrogen bond interactions. ${ }^{44}$ In the CMCS-TA-BDBA hydrogel, the peak ascribed to $-\mathrm{C}=\mathrm{O}$ vibration in $\mathrm{TA}$ shifted with a shoulder peak at $1699 \mathrm{~cm}^{-1}$ and the $-\mathrm{OH}$ bending vibration peaks shifted from 1609 to $1620 \mathrm{~cm}^{-1}$, which suggests that intermolecular hydrogen bonds are responsible for gel formation. ${ }^{24,45}$ Compared with the spectrum of TA, a new peak at $1731 \mathrm{~cm}^{-1}$, which was assigned to B-O stretching vibration, appeared in the spectrum of the CMCS-TA-BDBA hydrogel as a result of binding between the cis-diol moiety of TA to phenylboronic acid of BDBA. ${ }^{30,46}$ Upon TA binding, the $-\mathrm{OH}$ stretching modes shifted from $3296 \mathrm{~cm}^{-1}$ for free BDBA to $3481 \mathrm{~cm}^{-1}$ in CMCS-TA-BDBA (BDBA/TA 1:1) and $3554 \mathrm{~cm}^{-1}$ in 
CMCS-TA-BDBA (BDBA/TA 3:1). ${ }^{46}$ The smaller shift observed for CMCS-TABDBA (BDBA/TA 1:1) suggests less phenylboronate ester bonds in the system than those present in the hydrogels formed at a higher BDBA-to-TA ratio (BDBA/TA 3:1). From the FT-IR results (Figure S3), it could be concluded that hydrogen bonds were the major driving force for the gelation process. In addition, the dynamic covalent binding between BDBA and TA played a key role in the self-assembly of the CMCSTA-BDBA hydrogels.

In the tricomponent system, TA bound with CMCS and BDBA simultaneously. The influence of the BDBA-to-TA molar ratio on gel formation was explored (Figure 1d). At fixed concentrations of CMCS and TA, the $G^{\prime}$ and $G^{\prime \prime}$ values of the hydrogels initially increased with increasing BDBA-to-TA molar ratios up to a BDBA-to-TA molar ratio of 3:1, after which the $G^{\prime}$ and $G^{\prime \prime}$ values decreased. At a BDBA-to-TA molar ratio of $3: 1$, the $G^{\prime}$ and $G^{\prime \prime}$ of the gels were $2.5 \times 10^{4}$ and $3.0 \times 10^{3} \mathrm{~Pa}$, respectively, at an oscillatory frequency of $10 \mathrm{~Hz}$. In contrast, the $G^{\prime}$ and $G^{\prime \prime}$ values of the CMCS-TABDBA (BDBA/TA 4:1) hydrogels were $4.6 \times 10^{3}$ and $4.3 \times 10^{2} \mathrm{~Pa}$, respectively. This indicates that the cross-linking density reached maximum when the molar ratio of BDBA to TA was 3:1. At higher BDBA-to-TA molar ratios, possibly any excess BDBA molecules compete with CMCS to interact with the catechol or gallic groups of TA, which consequently disrupts the hydrogen bonds initially formed between CMCS and TA and therefore decreases the mechanical strength of the resulting hydrogel (Figure S4). In the CMCS-TA-BDBA hydrogel, the catechol-boric acid dynamic covalent bonds, hydrogen bonds, and a number of residual phenolic groups endow the obtained 
hydrogels with robustness of covalent bonds and the flexibility of noncovalent interactions. ${ }^{47-49}$ These interactions can be well balanced by tuning the ratios of CMCS/TA and BDBA/TA.

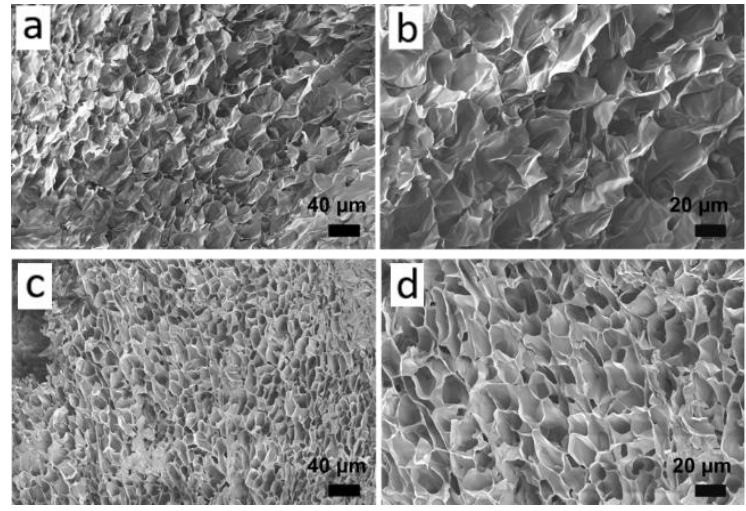

Figure 2. SEM images of the cross-sections of (a, b) CMCS-TA and (c, d) CMCS-TABDBA hydrogels.

As demonstrated above, the introduction of BDBA into the CMCS/TA system leads to formation of dynamic boronate ester bonds and influences the viscoelastic properties of the resulting hydrogels. The interior morphologies of the CMCS-TA and CMCSTA-BDBA hydrogels were observed by SEM. As shown in Figure 2, the hydrogels featured a porous $3 \mathrm{D}$ networks, with the porous structure of $40-80 \mu \mathrm{m}$ in diameter in the CMCS-TA hydrogel and 20-40 $\mu \mathrm{m}$ in diameter in the CMCS-TA-BDBA hydrogel. In addition, the CMCS-TA-BDBA hydrogel featured a more compact porous structure than the CMCS-TA hydrogel, which was effectively responsible for the higher mechanical strength of the CMCS-TA-BDBA hydrogels.

The injectability and sprayability of the hydrogels were investigated, as depicted in Figure 3. The gels were stained with rhodamine B for visualization purposes. To examine the injectability of the CMCS-TA-BDBA hydrogel, the solution of BDBA and 
CMCS (precursor solution A stained with rhodamine B) and solution of TA (precursor solution B) were filled into two separate syringes in a dual-syringe applicator (Figure 3a). When the plungers were pushed down, the two precursor solutions were extruded first through a "Y" shape connector and then through a narrow needle (18 G) to finally form a hydrogel in situ at room temperature $\left(25^{\circ} \mathrm{C}\right)$ (Figure $\left.3 \mathrm{~b}\right)$. To test the sprayability of the hydrogels, equal volumes of solution A and solution B were filled into separate sprayable bottles (Figure 3c). Precursor solution A was sprayed onto a petri dish followed by spraying of precursor solution B (TA solution). A fast-forming and selfsupported sprayable CMCS-TA-BDBA hydrogel was obtained (Figure 3d). This spray method is also suitable for the CMCS-TA hydrogels.

a

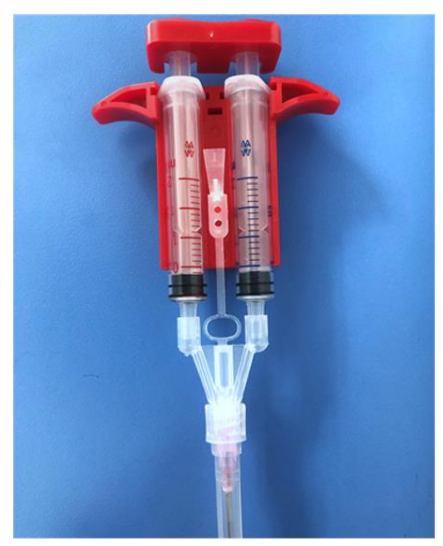

C

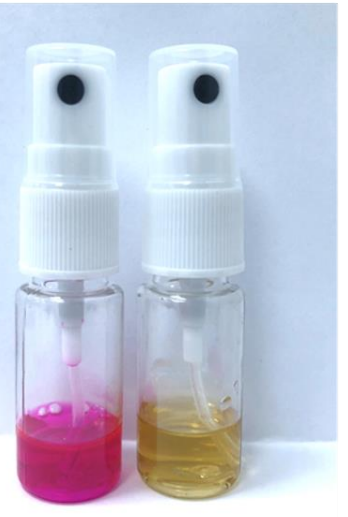

b

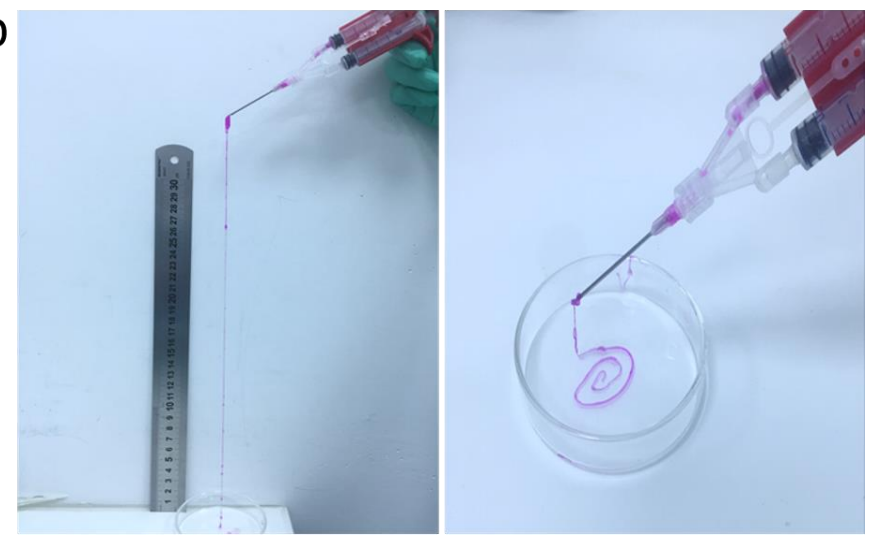

$d^{\prime}$
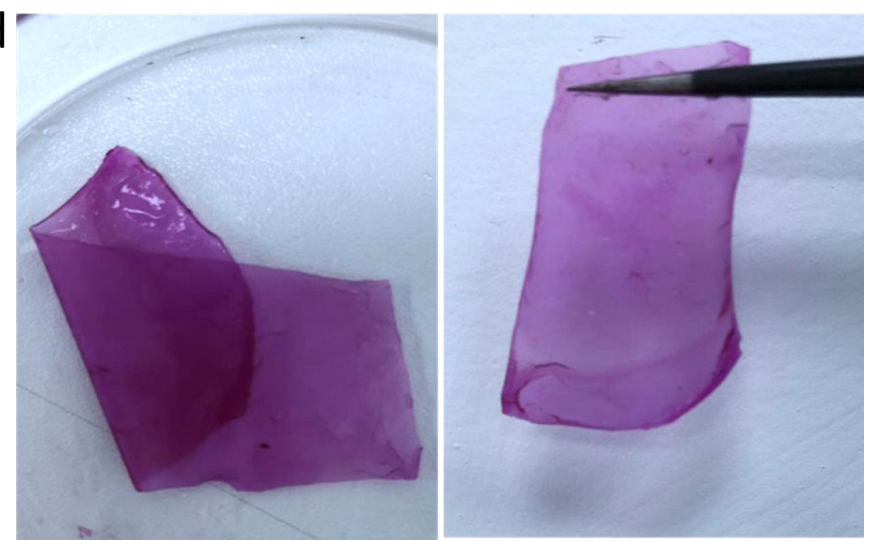

Figure 3. Photographs of (a) a double-syringe device, (b) the injectable CMCS-TABDBA hydrogel prepared using a dual-syringe applicator, (c) sprayable bottles 
containing precursor solutions $\mathrm{A}$ (BDBA and CMCS, stained with rhodamine $\mathrm{B}$ ) and $\mathrm{B}$ (TA), and (d) the self-supported sprayable hydrogel assembled by sequential spraying of solutions $\mathrm{A}$ and $\mathrm{B}$ on a petri dish.

The self-healing abilities of the CMCS-TA and CMCS-TA-BDBA hydrogels were qualitatively and quantitatively evaluated by a macroscopic recovery process (Figure 4) and dynamic oscillatory rheology (Figure 5), respectively. Hydrogels shown in Figure 4a stained with different colors were cut into halves and immediately brought into contact (Figure $4 b-d)$. The sectioned pieces could heal into an integral hydrogel and were then suspended under gravity after $60 \mathrm{~s}$ (Figure S5). Notably, the impregnated dye molecules continually spread after healing for $12 \mathrm{~h}$. Moreover, hydrogel strips stained with rhodamine B could pass through a $26 \mathrm{G}$ needle without clogging and gradually healed into a whole piece (Figure $4 \mathrm{e}-\mathrm{g}$ ). The hydrogels could also be used as "ink" for writing (Figure $4 \mathrm{~h}$ ). These results show the efficient self-healing process of the hydrogels prepared in the present study.

a

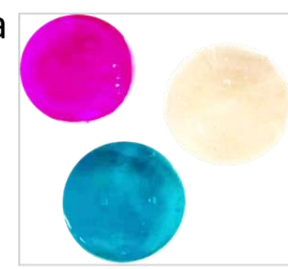

e

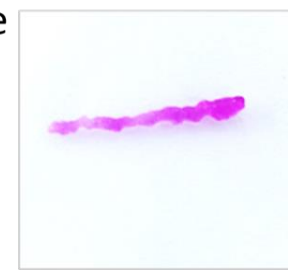

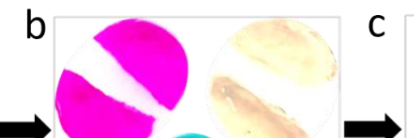


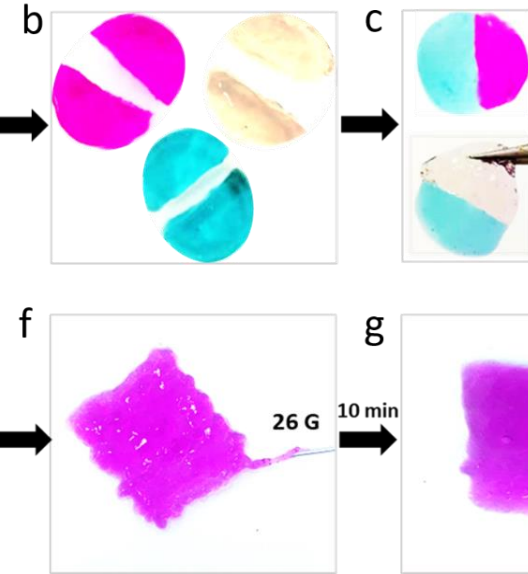

g

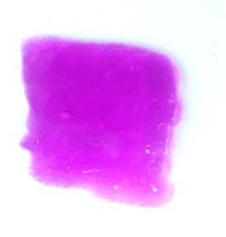

d

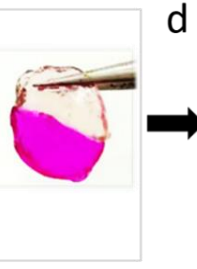

h

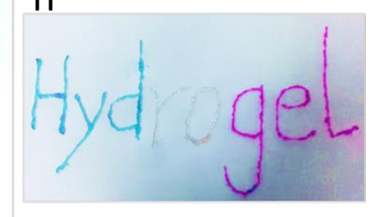

Figure 4. (a) Native (yellow) and stained CMCS-TA hydrogels. Pink and blue hydrogels were stained with rhodamine B and methylene blue, respectively. (b, c) 
Hydrogels were cut into halves and healed into integral hydrogel disks and suspended under gravity for 60 s. (d) Hydrogels healed and interconnected as a whole entity after $12 \mathrm{~h}$ at $25^{\circ} \mathrm{C}$. (e-g) Hydrogel strips passed through a $26 \mathrm{G}$ needle without clogging and healed gradually to a whole piece. (h) The word "hydrogel" written with hydrogel strips extruded from a syringe.

To further investigate the self-healing ability, the rheological properties of the hydrogels were determined. A stress sweep was used to rupture the hydrogels followed by an oscillatory time sweep to monitor recovery. At the beginning of the stress sweep, $G^{\prime}$ was higher than that of $G^{\prime \prime}$ under stress ranging from 1 to $400 \mathrm{~Pa}$, which indicates that the elastic component is dominant in the system (Figure 5a). ${ }^{38,48}$ The sol-to-gel transition point was defined as the intersection of the $G^{\prime}$ curve and $G^{\prime \prime}$ curve at $\sim 400$ $\mathrm{Pa}$, indicative of the onset of damage of the hydrogel network. ${ }^{44}$ Subsequently, the time sweep showed that the damaged hydrogel could recover its initial $G^{\prime}$ and $G^{\prime \prime}$ values within 30 s. Furthermore, the apparent viscosity $(\eta)$ decreased significantly with increasing shear rate (Figure 5b), which demonstrates the shear thinning behavior of the gels, ${ }^{50}$ thus demonstrating the potential application the hydrogels in in vivo injection. $^{48}$ 

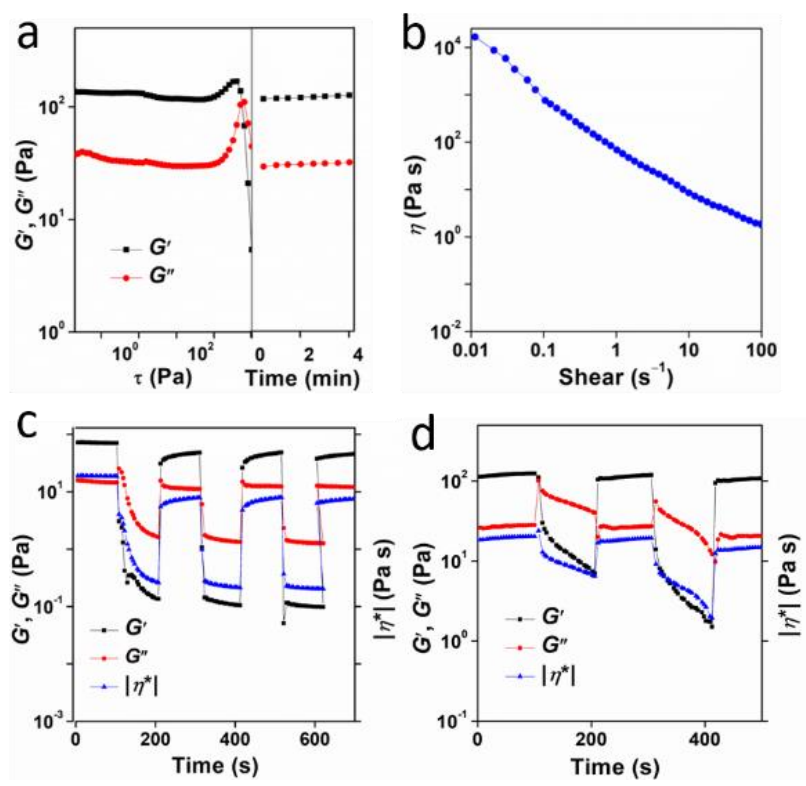

Figure 5. (a) Dynamic oscillatory rheology under applied shear stress ranging from 0.01 to $1000 \mathrm{~Pa}$ and the time recovery dependence of the CMCS-TA hydrogels at $1 \mathrm{~Hz}$. (b) Apparent viscosity $(\eta)$ rheology as a function of shear rate of the CMCS-TA hydrogels. Cyclic $G^{\prime}, G^{\prime \prime}$, and complex viscosity $\left|\eta^{*}\right|$ values of (c) CMCS-TA hydrogels and (d) CMCS-TA-BDBA hydrogels as a function of step stress from small stress (1 $\mathrm{Pa})$ to large stress (800 Pa) applied for fixed intervals of $100 \mathrm{~s}$.

In addition, dynamic oscillatory rheometry was used to determine the recovery rate. The hydrogels were repeatedly subjected to alternating cycles of applied low shear stress at $1 \mathrm{~Pa}$ and high stress at $800 \mathrm{~Pa}$ to break the gels periodically (Figure 5c). The gels were unaffected $\left(G^{\prime}>G^{\prime \prime}\right)$ at a low shear stress and became a sol $\left(G^{\prime}<G^{\prime \prime}\right)$ under a high shear stress. After reverting to the low strain, $G^{\prime}$ and $G^{\prime \prime}$ returned to their original values within less than $1 \mathrm{~s}$, demonstrating that the hydrogels can self-heal quickly and recover to a hydrogel phase over four cycles of breakage and recovery. ${ }^{50}$ The selfhealing of the CMCS-TA hydrogels is attributed to the formation of hydrogen bonds 
between CMCS and TA. Similarly, the CMCS-TA-BDBA hydrogel exhibited selfhealing behavior, which was due to the intermolecular hydrogen interactions as well as the dynamic boronate ester bonds (Figure 5d).

CMCS, TA, and BDBA have been proven to be biocompatible. ${ }^{13,20}$ Thus, the toxicity of the CMCS-TA and the CMCS-TA-BDBA hydrogels (BDBA/TA 1:1 and BDBA/TA 3:1) was examined using an MTT assay (Figure S6). The hydrogel degradation products (at $10 \mathrm{mg} \mathrm{mL}^{-1}$ ) resulted in approximate $80 \%$ cell viability, which improved to $90 \%$ at $5 \mathrm{mg} \mathrm{mL}{ }^{-1}$ and $\sim 100 \%$ cell viability at $2.5 \mathrm{mg} \mathrm{mL}^{-1}$, regardless of the ratio of BDBA and TA. The results demonstrate that the CMCS-TA-BDBA and CMCS-TA hydrogels possess low cytotoxicity.

Based on the biocompatibility and the gelation properties of the CMCS-TA-BDBA hydrogels, as well as the potential hemostatic activities of TA and CMCS, the antibleeding performance of the hydrogels was investigated. A mouse hepatic hemorrhage model was used to imitate severe trauma. The amount of blood loss experienced by a no treatment group (negative control), a gauze group (positive control), and a hydrogel group was measured. The mouse livers of all three groups at $180 \mathrm{~s}$ after injury bleeding are shown in Figure S7. Hydrogels were applied onto the bleeding sites through injection by a standard syringe and exhibited excellent hemostatic effects with a total bleeding amount of $55 \pm 19 \mathrm{mg}$, whereas the bleeding amounts obtained from the gauze-treated and no treatment groups were $180 \pm 20$ and $240 \pm 35 \mathrm{mg}$, respectively. A significant difference was found between the hydrogel group and the control groups $(P<0.001)$ (Figure 6a). It is hypothesized that the 
hydrogels could serve as an effective physical barrier for antibleeding (Figure 6b). TA is rich in gallic and catechol groups and CMCS contains abundant amine moieties, which synergistically contributed to the adhesion property of hydrogels under humid conditions for hemostasis. ${ }^{22}$ Moreover, the gallic and catechol groups of TA could react with the thiol, amine, imidazole residues of proteins and carbohydrates in the tissues. ${ }^{51-}$ ${ }^{53}$ As shown in Figure S8, broad bands of $-\mathrm{OH}$ stretching vibration appeared at $3481 \mathrm{~cm}^{-1}$ in the spectrum of the hydrogel while the $-\mathrm{OH}$ vibration characteristic peak shifted to $3280 \mathrm{~cm}^{-1}$ in the hydrogel treated with blood, which demonstrated the existence of hydrogen bonds between hydrogel and blood components. The $-\mathrm{C}=\mathrm{O}$ vibration characteristic peak in hydrogel was observed at $1710 \mathrm{~cm}^{-1}$ and shifted to $1654 \mathrm{~cm}^{-1}$ when the hydrogel interacted with blood, which suggested that the carbonyl groups were involved in the hydrogen bond interactions. Therefore, strong interactions (e.g., hydrogen bonds, cation- $\pi$ interactions and covalent bonds) between CMCS, TA, and blood components resulted in fast blood clotting (Figure 6c). ${ }^{23,54,55}$ The in vivo hemostatic experiments demonstrated that the CMCSTA-BDBA hydrogel had significant potential as local hemostatic agents, which could significantly reduce the bleeding amount. 

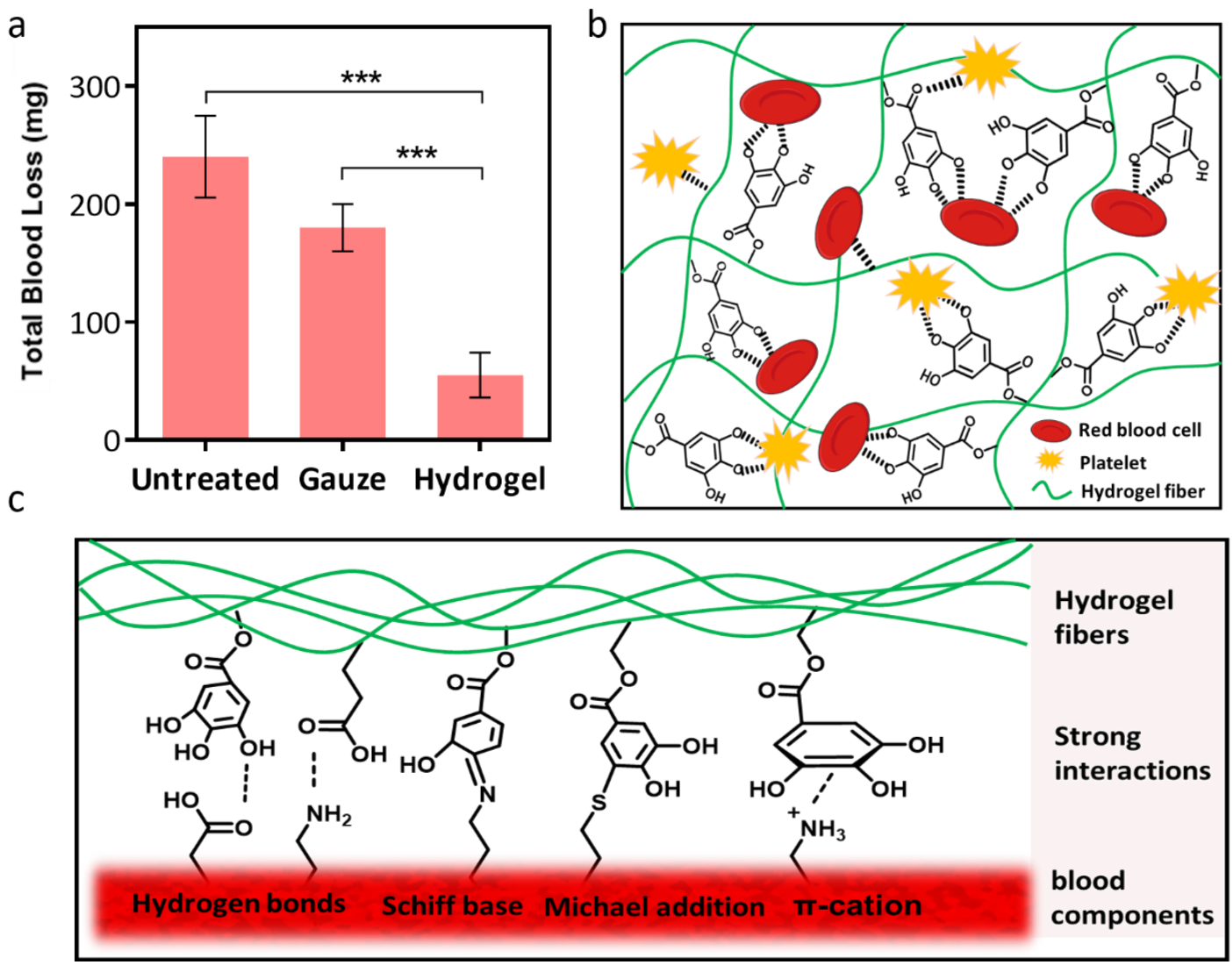

Figure 6. (a) Total amount of bleeding at $180 \mathrm{~s}$ after the injury $(n=3, * * * p<0.001)$.

(b) Scheme of the hydrogel, as a hemostatic agent, interacting with red blood cells and platelets. (c) Physical interactions and interfacial covalent cross-linking between the functional groups of gelators and blood.

\section{CONCLUSIONS}

In summary, we have developed a facile method to prepare TA/biomacromoleculebased hydrogels with potential for hemostasis. The intermolecular hydrogen bonds and dynamic boronate linkages are responsible for gel formation. The interactions can be balanced by tuning the weight ratios of $\mathrm{CMCS} / \mathrm{TA}$ and TA/BDBA, which is key for the construction of supramolecular hydrogels. The CMCS-TA-BDBA hydrogels benefit from ease and scalability of the assembly process and flexibility of injection and 
spraying. The reported method provides a platform for the rapid assembly of polyphenol-based hydrogels with potential applications in hemostasis, wound closure, tissue sealant, and drug delivery.

\section{ASSOCIATED CONTENT}

\section{Supporting Information}

Photographs of the solution, hydrogel, and coacervation of the CMCS-TA mixture, scheme of the formation of dynamic boronate ester bonds between BDBA and TA, characterizations (FT-IR, cytotoxicity) of CMCS-TA and AMCS-TA-BDBA hydrogel, photographs of the bleeding of the injured mouse liver after injury with and without treatment with hydrogels.

\section{AUTHOR INFORMATION}

\section{Corresponding Authors}

*E-mail: jwcui@sdu.edu.cn.

\section{ORCID}

Jiwei Cui: 0000-0003-1018-4336

\section{Notes}

The authors declare no competing financial interest.

\section{ACKNOWLEDGEMENT}

This research was funded by the National Natural Science Foundation of China (J.C., 21872085; Q.D., 21802088) and Natural Science Foundation of Shandong Province (H.G., ZR2019BB056; Q.D., ZR2018BB036).

\section{REFERENCES}


(1) Yu, L.; Ding, J. Injectable Hydrogels as Unique Biomedical Materials. Chem. Soc. Rev. 2008, 37, 1473-1481.

(2) Yu, S.; He, C.; Chen, X. Injectable Hydrogels as Unique Platforms for Local Chemotherapeutics-Based Combination Antitumor Therapy. Macromol. Biosci. 2018, $18, \mathrm{e} 1800240$.

(3) Yuk, H.; Lu, B.; Zhao, X. Hydrogel Bioelectronics. Chem. Soc. Rev., 2019, 48, 1642.

(4) Du, X.; Zhou, J.; Shi, J.; Xu, B. Supramolecular Hydrogelators and Hydrogels: From Soft Matter to Molecular Biomaterials. Chem. Rev. 2015, 115, 13165-13307.

(5) Dimatteo, R.; Darling, N. J.; Segura, T. In Situ Forming Injectable Hydrogels for Drug Delivery and Wound Repair. Adv. Drug Delivery Rev. 2018, 127, 167-184.

(6) Li, Y.; Rodrigues, J.; Tomas, H. Injectable and Biodegradable Hydrogels: Gelation, Biodegradation and Biomedical Applications. Chem. Soc. Rev. 2012, 41, 2193-2221.

(7) Jeong, Y.; Jo, Y. K.; Kim, B. J.; Yang, B.; Joo, K. I.; Cha, H. J. Sprayable Adhesive Nanotherapeutics: Mussel-Protein-Based Nanoparticles for Highly Efficient Locoregional Cancer Therapy. ACS Nano 2018, 12, 8909-8919.

(8) Chen, Q.; Wang, C.; Zhang, X.; Chen, G.; Hu, Q.; Li, H.; Wang, J.; Wen, D.; Zhang, Y.; Lu, Y.; Yang, G.; Jiang, C.; Wang, J.; Dotti, G.; Gu, Z. In Situ Sprayed Bioresponsive Immunotherapeutic Gel for Post-Surgical Cancer Treatment. Nat. Nanotechnol. 2019, 14, 89-97.

(9) Wang, X.; Wang, C.; Wang, X.; Wang, Y.; Zhang, Q.; Cheng, Y. A Polydopamine Nanoparticle-Knotted Poly(ethylene Glycol) Hydrogel for On-Demand Drug Delivery and Chemo-Photothermal Therapy. Chem. Mater. 2017, 29, 1370-1376.

(10) Azevedo, S.; Costa, A. M. S.; Andersen, A.; Choi, I. S.; Birkedal, H.; Mano, J. F. Bioinspired Ultratough Hydrogel with Fast Recovery, Self-Healing, Injectability and Cytocompatibility. Adv. Mater. 2017, 29, 1700759.

(11) Cao, L.; Cao, B.; Lu, C.; Wang, G.; Yu, L.; Ding, J. An Injectable Hydrogel Formed by In Situ Cross-Linking of Glycol Chitosan and Multi-Benzaldehyde Functionalized PEG Analogues for Cartilage Tissue Engineering. J. Mater. Chem. B 2015, 3, 1268-1280.

(12) Deng, H.; Dong, A.; Song, J.; Chen, X. Injectable Thermosensitive Hydrogel Systems Based on Functional PEG/PCL Block Polymer for Local Drug Delivery. $J$. Controlled Release 2019, 297, 60-70.

(13) Ghobril, C.; Grinstaff, M. W. The Chemistry and Engineering of Polymeric Hydrogel Adhesives for Wound Closure: A Tutorial. Chem. Soc. Rev. 2015, 44, 1820 1835.

(14) Qua, J.; Zhao, X.; Liang, Y.; Xu, Y.; Ma, P. X.; Guo, B. Degradable Conductive Injectable Hydrogels as Novel Antibacterial, Antioxidant Wound Dressings for Wound Healing. Chem. Eng. J. 2019, 362, 548.

(15) Chen, T.; Chen, Y.; Rehman, H. U.; Chen, Z.; Yang, Z.; Wang, M.; Li, H.; Liu, H. Ultratough, Self-Healing, and Tissue-Adhesive Hydrogel for Wound Dressing. ACS Appl. Mater. Interfaces 2018, 10, 33523-33531.

(16) Hu, B.; Shen, Y.; Adamcik, J.; Fischer, P.; Schneider, M.; Loessner, M. J.; Mezzenga, R. Polyphenol-Binding Amyloid Fibrils Self-Assemble into Reversible Hydrogels with Antibacterial Activity. ACS Nano 2018, 12, 3385-3396. 
(17) Ejima, H.; Richardson, J. J.; Liang, K.; Best, J. P.; van Koeverden, M. P.; Such, G. K.; Cui, J.; Caruso, F. One-Step Assembly of Coordination Complexes for Versatile Film and Particle Engineering. Science 2013, 341, 154-157.

(18) Sileika, T. S.; Barrett, D. G.; Zhang, R.; Lau, K. H.; Messersmith, P. B. Colorless Multifunctional Coatings Inspired by Polyphenols Found in Tea, Chocolate, and Wine. Angew. Chem. Int. Ed. 2013, 52, 10766-10770.

(19) Zhong, Q. Z.; Li, S.; Chen, J.; Xie, K.; Pan, S.; Richardson, J. J.; Caruso, F. Oxidation-Mediated Kinetic Strategies for Engineering Metal-Phenolic Networks. Angew. Chem. Int. Ed. 2019, 58, 12563-12568.

(20) Zhang, L.; Wan, S. S.; Li, C. X.; Xu, L.; Cheng, H.; Zhang, X. Z. An Adenosine Triphosphate-Responsive Autocatalytic Fenton Nanoparticle for Tumor Ablation with Self-Supplied $\mathrm{H}_{2} \mathrm{O}_{2}$ and Acceleration of $\mathrm{Fe}(\mathrm{III}) / \mathrm{Fe}(\mathrm{II})$ Conversion. Nano Lett. 2018, 18, 7609-7618.

(21) Wang, C.; Zhou, H.; Niu, H.; Ma, X.; Yuan, Y.; Hong, H.; Liu, C. Tannic AcidLoaded Mesoporous Silica for Rapid Hemostasis and Antibacterial Activity. Biomater. Sci. 2018, 6, 3318-3331.

(22) Li, N.; Yang, X.; Liu, W.; Xi, G.; Wang, M.; Liang, B.; Ma, Z.; Feng, Y.; Chen, H.; Shi, C. Tannic Acid Cross-Linked Polysaccharide-Based Multifunctional Hemostatic Microparticles for the Regulation of Rapid Wound Healing. Macromol. Biosci. 2018, $18, \mathrm{e} 1800209$.

(23) Chen, J.; Cheng, W.; Chen, S.; Xu, W.; Lin, J.; Liu, H.; Chen, Q. UrushiolFunctionalized Mesoporous Silica Nanoparticles and Their Self-Assembly into a Janus Membrane as a Highly Efficient Hemostatic Material. Nanoscale 2018, 10, 2281822829.

(24) Fan, H.; Wang, L.; Feng, X.; Bu, Y.; Wu, D.; Jin, Z. Supramolecular Hydrogel Formation Based on Tannic Acid. Macromolecules 2017, 50, 666-676.

(25) Kim, K.; Shin, M.; Koh, M.-Y.; Ryu, J. H.; Lee, M. S.; Hong, S.; Lee, H. TAPE: A Medical Adhesive Inspired by a Ubiquitous Compound in Plants. Adv. Funct. Mater. 2015, 25, 2402-2410.

(26) Zhou, L.; Fan, L.; Yi, X.; Zhou, Z.; Liu, C.; Fu, R.; Dai, C.; Wang, Z.; Chen, X.; Yu, P.; Chen, D.; Tan, G.; Wang, Q.; Ning, C. Soft Conducting Polymer Hydrogels Cross-Linked and Doped by Tannic Acid for Spinal Cord Injury Repair. ACS Nano 2018 , 12, 10957-10967.

(27) Shukla, A.; Fang, J. C.; Puranam, S.; Jensen, F. R.; Hammond, P. T. Hemostatic Multilayer Coatings. Adv. Mater. 2012, 24, 492-496.

(28) Guo, J.; Sun, W.; Kim, J. P.; Lu, X.; Li, Q.; Lin, M.; Mrowczynski, O.; Rizk, E. B.; Cheng, J.; Qian, G.; Yang, J. Development of Tannin-Inspired Antimicrobial Bioadhesives. Acta Biomater. 2018, 72, 35-44.

(29) Shi, L.; Ding, P.; Wang, Y.; Zhang, Y.; Ossipov, D.; Hilborn, J. Self-Healing Polymeric Hydrogel Formed by Metal-Ligand Coordination Assembly: Design, Fabrication, and Biomedical Applications. Macromol. Rapid Commun. 2019, 40, e1800837.

(30) Guo, J.; Sun, H.; Alt, K.; Tardy, B. L.; Richardson, J. J.; Suma, T.; Ejima, H.; Cui, J.; Hagemeyer, C. E.; Caruso, F. Boronate-Phenolic Network Capsules with Dual 
Response to Acidic pH and Cis-Diols. Adv. Healthcare Mater. 2015, 4, 1796-1801.

(31) Rahim, M. A.; Kristufek, S. L.; Pan, S.; Richardson, J. J.; Caruso, F. Phenolic Building Blocks for the Assembly of Functional Materials. Angew. Chem. Int. Ed. 2018, $57,2-26$.

(32) Ejima, H.; Richardson, J. J.; Caruso, F. Metal-Phenolic Networks as a Versatile Platform to Engineer Nanomaterials and Biointerfaces. Nano Today 2017, 12, 136-148.

(33) Lomova, M. V.; Brichkina, A. I.; Kiryukhin, M. V.; Vasina, E. N.; Pavlov, A. M.; Gorin, D. A.; Sukhorukov, G. B.; Antipina, M. N. Multilayer Capsules of Bovine Serum Albumin and Tannic Acid for Controlled Release by Enzymatic Degradation. ACS Appl. Mater. Interfaces 2015, 7, 11732-11740.

(34) Chen, J.; Ratnayaka, S.; Alford, A.; Kozlovskaya, V.; Liu, F.; Xue, B.; Hoyt, K.; Kharlampieva, E. Theranostic Multilayer Capsules for Ultrasound Imaging and Guided Drug Delivery. ACS Nano 2017, 11, 3135-3146.

(35) Krogsgaard, M.; Andersen, A.; Birkedal, H. Gels and Threads: Mussel-Inspired One-Pot Route to Advanced Responsive Materials. Chem. Commun. 2014, 50, 1327813281.

(36) Wang, Y.; Kim, K.; Lee, M. S.; Lee, H. Hemostatic Ability of Chitosan-Phosphate Inspired by Coagulation Mechanisms of Platelet Polyphosphates. Macromol. Biosci. 2018, 18, e1700378.

(37) Shin, M.; Ryu, J. H.; Park, J. P.; Kim, K.; Yang, J. W.; Lee, H. DNA/Tannic Acid Hybrid Gel Exhibiting Biodegradability, Extensibility, Tissue Adhesiveness, and Hemostatic Ability. Adv. Funct. Mater. 2015, 25, 1270-1278.

(38) Feng, C.; Wang, Z.; Jiang, C.; Kong, M.; Zhou, X.; Li, Y.; Cheng, X.; Chen, X. Chitosan/O-Carboxymethyl Chitosan Nanoparticles for Efficient and Safe Oral Anticancer Drug Delivery: In Vitro and In Vivo Evaluation. Int. J. Pharm. 2013, 457, $158-167$.

(39) Geng, H.; Ye, L.; Zhang, A. Y.; Li, J.; Feng, Z. G. Low-Molecular-Weight Organoand Hydrogelators Based on Cyclo(L-Lys- L-Glu). Langmuir 2016, 32, 4586-4594.

(40) Xu, H.; Nishida, J.; Ma, W.; Wu, H.; Kobayashi, M.; Otsuka, H.; Takahara, A. Competition between Oxidation and Coordination in Cross-Linking of Polystyrene Copolymer Containing Catechol Groups. ACS Macro. Lett. 2012, 1, 457-460.

(41) Schneider, A.; Hemmerle, J.; Allais, M.; Didierjean, J.; Michel, M.; d'Ischia, M.; Ball, V. Boric Acid as an Efficient Agent for the Control of Polydopamine SelfAssembly and Surface Properties. ACS Appl. Mater. Inter. 2018, 10, 7574-7580.

(42) He, L.; Fullenkamp, D. E.; Rivera, J. G.; Messersmith, P. B. pH Responsive SelfHealing Hydrogels Formed by Boronate-Catechol Complexation. Chem. Commun. 2011, 47, 7497-7499.

(43) Matsumoto, A.; Tanaka, M.; Matsumoto, H.; Ochi, K.; Moro-oka, Y.; Kuwata, H.; Yamada, H.; Shirakawa, I.; Miyazawa, T.; Ishii, H.; Kataoka, K.; Ogawa, Y.; Miyahara, Y.; Suganami, T. Synthetic "Smart Gel” Provides Glucose-Responsive Insulin Delivery in Diabetic Mice. Sci. Adv. 2017, 3, eaaq0723.

(44) Shin, M.; Kim, K.; Shim, W.; Yang, J. W.; Lee, H. Tannic Acid as a Degradable Mucoadhesive Compound. ACS Biomater. Sci. Eng. 2016, 2, 687-696.

(45) Zhao, L.; Zou, Q.; Yan, X. Self-Assembling Peptide-Based Nanoarchitectonics. 
Bull. Chem. Soc. Jpn. 2019, 92, 70.

(46) Vahlberg, C.; Linares, M.; Norman, P.; Uvdal, K. Phenylboronic Ester- and Phenylboronic Acid-Terminated Alkanethiols on Gold Surfaces. J. Phys. Chem. C 2011, 116, 796-806.

(47) Guo, R.; Su, Q.; Zhang, J.; Dong, A.; Lin, C.; Zhang, J. Facile Access to Multisensitive and Self-Healing Hydrogels with Reversible and Dynamic Boronic Ester and Disulfide Linkages. Biomacromolecules 2017, 18, 1356-1364.

(48) Zhang, Z.; Wang, X.; Wang, Y.; Hao, J. Rapid-Forming and Self-Healing AgaroseBased Hydrogels for Tissue. Biomacromolecules 2018, 19, 980-988.

(49) Huang, W.; Wang, Y.; Chen, Y.; Zhao, Y.; Zhang, Q.; Zheng, X.; Chen, L.; Zhang, L. Strong and Rapidly Self-Healing Hydrogels: Potential Hemostatic Materials. $A d v$. Healthcare Mater. 2016, 5, 2813-2822.

(50) Gao, Z.; Sui, J.; Xie, X.; Li, X.; Song, S.; Zhang, H.; Hu, Y.; Hong, Y.; Wang, X.; Cui, J.; Hao, J. Metal-Organic Gels of Simple Chemicals and Their High Efficacy in Removing Arsenic(V) in Water. AIChE J. 2018, 64, 3719-3727.

(51) Chen, W.; Wang, R.; Xu, T.; Ma, X.; Yao, Z.; Chi, B.; Xu, H. A Mussel-Inspired Poly $(\gamma$-Glutamic Acid) Tissue Adhesive with High Wet Strength for Wound Closure. $J$. Mater. Chem. B 2017, 5, 5668-5678.

(52) Wang, R.; Li, J.; Chen, W.; Xu, T.; Yun, S.; Xu, Z.; Xu, Z.; Sato, T.; Chi, B.; Xu, H. A Biomimetic Mussel-Inspired $\varepsilon$-Poly-1-lysine Hydrogel with Robust Tissue-Anchor and Anti-Infection Capacity. Adv. Funct. Mater. 2017, 27, 1604894.

(53) Cho, J. H.; Lee, J. S.; Shin, J.; Jeon, J. J.; An, S.; Choi, Y. S.; Cho, S-W. AscidianInspired Fast-Forming Hydrogel System for Versatile Biomedical Applications: Pyrogallol Chemistry for Dual Modes of Crosslinking Mechanism. Adv. Funct. Mater. 2018, 28, 1705244.

(54) Lu, D.; Wang, H.; Li, T.; Li, Y.; Wang, X.; Niu, P.; Guo, H.; Sun, S.; Wang, X.; Guan, X.; Ma, H.; Lei, Z. Versatile Surgical Adhesive and Hemostatic Materials: Synthesis, Properties, and Application of Thermoresponsive Polypeptides. Chem. Mater. 2017, 29, 5493.

(55) Hong, S.; Wang, Y.; Park, S. Y.; Lee, H. Progressive Fuzzy Cation- $\pi$ Assembly of Biological Catecholamines. Sci. Adv. 2018, 4, eaat7457. 
Graphical Table of Contents:
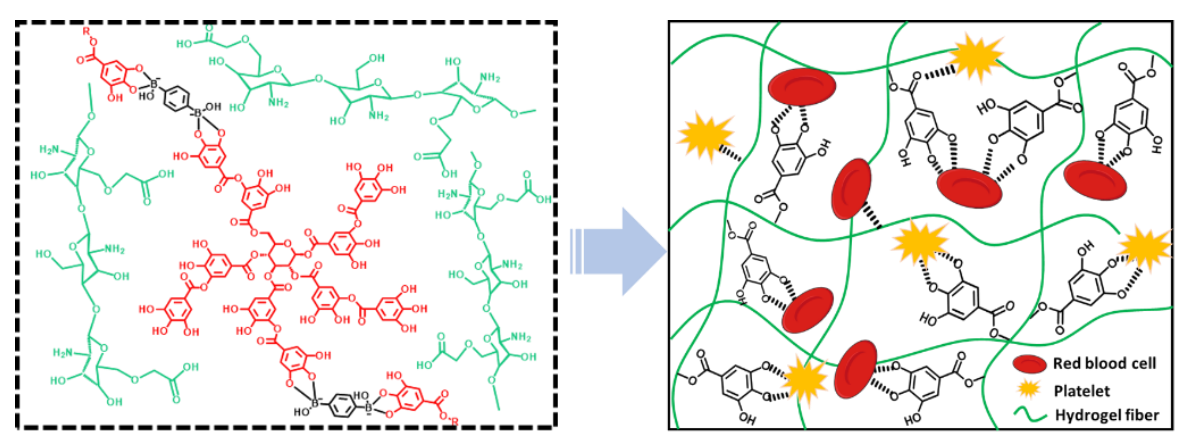